\title{
Results of Geoinformation Technologies and Unmanned Aerial Vehicle Application for Maroc Locust Larvae Identification
}

ISSN: 2637-7659

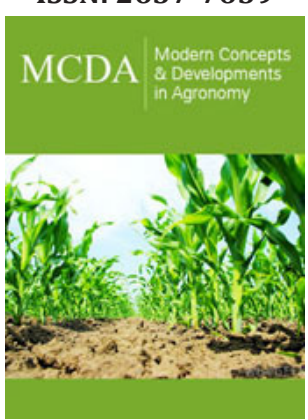

*Corresponding author: Uspanov AM, Kazakh Research Institute for Plant protection and Quarantine after ZhazkenZhiembayev, Almaty, Republic of Kazakhstan

\section{Submission: 些- May11, 2020}

Published: 毕 November 11, 2020

Volume 7 - Issue 4

How to cite this article: Boltayev MD, Kambulin VY, NiyazbekovZhB, Yessimov UO, Bashkarayev NA and Uspanov AM. Results of Geoinformation Technologies and Unmanned Aerial Vehicle Application for Maroc Locust Larvae Identification. Mod Concep Dev Agrono. 7(4). MCDA. 000666. 2020. DOI: 10.31031/MCDA.2020.07.000666

Copyright@ Uspanov AM, This article is distributed under the terms of the Creative Commons Attribution 4.0 International License, which permits unrestricted use and redistribution provided that the original author and source are credited.

\section{Boltayev MD, Kambulin VY, Niyazbekov ZhB, Yessimov UO, Bashkarayev NA and} Uspanov AM*

Kazakh Research Institute for Plant protection and Quarantine after ZhazkenZhiembayev Almaty, Republic of Kazakhstan

\section{Annotation}

The possibility of maroc locust identification with the appliance of unmanned aerial vehicle has been studied. VARI index application allowed to identify the areas of damaged vegetation by locust. As the result of combining the processed data of remote sensing, layers of geo information system and field data, the thematic digital maps of maroc locust spread and population are taken.

Abbreviations: GIT: Geoinformation Technologies; UAVs: SmallSized Unmanned Aerial Vehicles VARI: Visible Atmospherically Resistant Index

\section{Introduction}

Nowadays, it is extremely necessary to get exact and reliable information about agricultural fields' phytosanitary condition and usage estimation both, to support the activity of agro industrial enterprises and environmental control. The improvement of phytosanitary monitoring methods and technologies are the actual and priority tasks for agriculture of the Republic of Kazakhstan.

Maroc locust (Dociostaurus maroccanus Thunb.) is an aggressive specie, that damages all types of crops including young trees and vineyards. The range of this specie covers territories of Turkestan, Zhambyl and Almaty regions, and the population of it can reach up to 3 thousand individuals per square meter in auspicious years.

Today, the geoinformation technologies (GIT) are actively developing in the world and used to solve a lot of various tasks including phytosanitary safety. Small-sized unmanned aerial vehicles (UAVs) are widely used in the world. According to expert's estimation, the number of commercial UAVs in the world market will reach 200000 in 2020 [1].

The challenge of UAVs application as a new photometrical tool is the weakness of two traditional ways of obtaining the remote sensing data using satellite (space survey) and manned aerial vehicle (aerial photography). Space survey allows to get images with maximum public resolution of $0,5 \mathrm{~m}$., which is not enough for large scale mapping. Traditional aerial photography, which is carried out using airplanes or helicopters, requires high economic costs for maintenance, fuel, security, etc., which leads to higher cost of the final product [2].

The experience of using drones for agricultural aerial photography showed the superiority of using UAVs helicopter (multi-rotor) over aircraft-type drones for agriculture that can solve many problems faster and cheaper than satellites. It means the on-time works, the same day shooting will help to undertake prompt actions on actual issues. Moreover, changes in the situation over time will also be promptly reflected [3].

\section{Material and Methods}

Kazakh Research Institute for Plant protection and Quarantine after. Zh. Zhiembayev makes research for maroc locust survey methods improvement using UAVs and GIS under the 
Ministry of Agriculture of the Republic of Kazakhstan since 2018 in the frames of the budget program for 2018-2020 "Improvement of knowledge and scientific research availability".

Based on the selected UAVs (quad and hexacopter) and photo equipment (RGB cameras with a resolution of over 16 megapixels and Tetracam IR cameras), optimal meteorological, flightnavigation, optical and identification parameters were developed in the system of technology for remote phytosanitary monitoring of swarm locust focus.

Research main concept is to conduct simultaneous survey of young age pests' swarms by field monitoring and UAVs route flights over the points for comparing and the possible identification of it in the images.

\section{Research Results and Discussion}

Maroc locusts survey methodology development using UAVs was carried out on arid pastures in Turkestan region. Aerial searches were carried out in three stages:

A. preparatory stage contained cameras calibration, georeferencing of the characteristic's points, aerial survey design.

B. Stage of flight survey consisted of aerial survey design, UAVs flight routes design and scheduled flights.

C. Stage of office operation is an aerial pictures processing and mosaic creation.

The survey of maroc locust territory was carried out using DJI Phantom 4 PRO quadcopter and 3DR Solo. At survey moment maroc locust was mainly in 2-3 age phase. The number of swarm individuals varied from 100 to 1000 per square meter. The method of straight parallel flight was used for area (test-polygon) aerial photography. This method covers the maximum width of the UAVs camera field view at a fixed height and constant speed of flight.

Several hundred pictures were obtained. The geometrical and color-corrected orthophoto map had been created as a result of all images combining. Based on the obtained images, field and visual decryption of aerial photographs was carried out, direct decoding signs of pests (shape, size, tone, swarms image structure) and indirect signs (life activity traces, changes in vegetation properties etc.) were established.

VARI index was estimated with the help of QGIS charge-free software. The use of mentioned index allowed to identify: red-badly damaged areas by locust, from green to blue-undamaged areas.
Images taken in the infrared range by UAV mounted camera were processed by ENVI 5.1 software. According to the infrared channel, IsoData method classification was carried out and areas with different vegetation state were identified. So, in areas where swarms of locusts were discovered, vegetation was practically absent or looked more depressed.

The process of image classification for identifying maroc locust swarms based on Drone Deploy ready-made automated solutions (cloud service and application for processing aerial photography data from UAVs, 2D high-resolution mapping, terrain 3D models).

Plant health algorithm such as VARI (Visible Atmospherically Resistant Index) compares the light proportions captured in different ranges (red, green, blue) to calculate the numerical values for each pixel or area of a given map obtained from an unmanned aerial vehicle.

It is found that healthy plants reflect light differently than depressed ones. Healthier plants tend to reflect more green light than red.

Consequently, the use of mentioned index proved the possibility of locust detection by indirect signs: red areas are badly damaged by the locusts, from green to blue - the undamaged area.

The development of digital thematic maps of maroc locusts spread and population was carried out on the basis of a geographic information system (GIS) created as part of this project. Cloud-free satellite images of medium spatial resolution over the vegetation season served as the basis for thematic maps of the soil and vegetation cover. Topographic vector data (district boundaries, roads, hydrographic network, settlements), field survey data (locust detection points) are loaded to GIS as layers. As the result of combining the processed data of remote sensing, layers of geo information system and field data, the thematic digital maps of maroc locusts spread and population are taken.

\section{Conclusion}

It is possible to detect vegetation damaged areas caused by locusts using satellite data, UAVs aerial photographs in combination with field data. Space imagery helps to make large scale identification of young age maroc locust larvae in terms of wide areas.

\section{References}

1. UAVs marketing research (2014) Agency for Industrial Information.

2. Zinchenko ON (2011) Unmanned aerial vehicle: application for aerial survey in mapping//Rakurs: [electronic resource], Moscow, Russia.

3. (2020) UAVs in agriculture [electronic resource].

For possible submissions Click below: 